\title{
Evidence for a T Tauri Phase in Young Brown Dwarfs
}

\author{
Ray Jayawardhana \\ Department of Astronomy, University of Michigan, 830 Dennison, Ann Arbor, MI 48109 \\ Subhanjoy Mohanty \\ Harvard-Smithsonian Center for Astrophysics, 60 Garden St., Cambridge, MA 02138 \\ and \\ Gibor Basri \\ Department of Astronomy, University of California at Berkeley, Berkeley, CA 94720
}

\begin{abstract}
As part of a multi-faceted program to investigate the origin and early evolution of sub-stellar objects, we present high-resolution Keck optical spectra of 14 very low mass sources in the IC 348 young cluster and the Taurus star-forming cloud. All of our targets, which span a range of spectral types from M5 to M8, exhibit moderate to very strong $\mathrm{H} \alpha$ emission. In half of the IC 348 objects, the $\mathrm{H} \alpha$ profiles are broad and asymmetric, indicative of on-going accretion. Of these, IC348-355 (M8) is the lowest mass object to date to show accretion-like $\mathrm{H} \alpha$. Three of our $\sim \mathrm{M} 6$ IC 348 targets with broad $\mathrm{H} \alpha$ also harbor broad OI

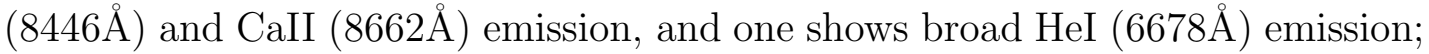
these features are usually seen in strongly accreting classical $\mathrm{T}$ Tauri stars. We find that in very low mass accretors, the $\mathrm{H} \alpha$ profile may be somewhat narrower than that in higher mass stars. We propose that low accretion rates combined with small infall velocities at very low masses can conspire to produce this effect. In the non-accretors in our sample, $\mathrm{H} \alpha$ emission is commensurate with, or higher than, saturated levels in field M dwarfs of similar spectral type. Our results constitute the most compelling evidence to date that young brown dwarfs undergo a $\mathrm{T}$ Tauri-like accretion phase similar to that in stars. This is consistent with a common origin for most low-mass stars, brown dwarfs and isolated planetary mass objects.
\end{abstract}

Subject headings: stars: low mass, brown dwarfs - stars: pre-main-sequence circumstellar matter - planetary systems - stars: formation - techniques: spectroscopic 


\section{Introduction}

Brown dwarfs, which straddle the mass range between stars and planets, appear to be common both in the field and in star-forming regions. Their ubiquity makes the question of their origin an important one, both for our understanding of brown dwarfs themselves as well as for theories on the formation of stars and planets.

In the standard framework, a low-mass star forms out of a collapsing cloud fragment, and goes through a "T Tauri phase", during which it accretes material from a surrounding disk, before arriving on the main sequence. There is ample observational evidence now to support many key aspects of this picture for young solar-mass stars. One of the defining characteristics of the $\mathrm{T}$ Tauri phase is a rich array of emission lines in the spectra, of which $\mathrm{H} \alpha$ is usually the most prominent (Joy 1945). The broad, asymmetric H $\alpha$ line profiles, seen in "classical" T Tauri stars, are now thought to arise in the infall region, as are many of the metallic emission lines (Muzerolle, Hartmann \& Calvet 1998 and references therein).

Whether the same scenario holds for objects at and below the sub-stellar limit is an

open question. It has been suggested, most recently by Padoan \& Nordlund (2003), that brown dwarfs form in the same way as more massive stars, via 'turbulent fragmentation'. Reipurth \& Clarke (2001) proposed an alternate scenario, which has been further explored through numerical simulations by Bate et al. (2002; 2003): in their model, brown dwarfs (and presumably isolated planetary mass objects or "planemos") are stellar embryos, ejected from newborn multiple systems before they can accrete sufficient mass to eventually fuse hydrogen. In this scenario, a stellar embryo competes with its siblings in order to accrete infalling matter, and the one that grows slowest is most likely to be ejected through dynamical interactions. A key prediction of Reipurth \& Clarke (2001) is that "sub-stellar equivalents to the classical T Tauri stars should be rather short-lived" because the ejection process limits the amount of gas brought along in a disk.

Studies of young sub-stellar objects could provide valuable clues to distinguish between these formation mechanisms. Therefore, we have undertaken a multi-faceted study of very low mass (VLM) objects in star-forming regions and their immediate circumstellar environment. One of our key goals is to investigate whether some or all young brown dwarfs undergo a T Tauri-like phase, and if so how long that phase lasts.

This paper reports on a search for accretion signatures in high-resolution spectra of 14 objects near and below the sub-stellar boundary in IC $348(\sim 320$ pc $)$ and Taurus $(\sim 150$ pc). Our targets consist of M5-M8 sources in IC 348 from Luhman (1999) and the recently 
identified M7-M8 brown dwarfs in Taurus from Martín et al. (2001) ${ }^{1}$. Combined with the spectra of 15 additional sources in the Upper Scorpius and $\rho$ Ophiuchus star-forming regions (Jayawardhana, Mohanty \& Basri 2002), we now have a substantial sample to characterize disk accretion in young brown dwarfs.

In a subsequent paper, we will use these optical spectra to derive temperatures, gravities and masses for the current sample, as we did for Upper Sco and $\rho$ Oph (Mohanty et al. 2003a, 2003b). For now, we assume an age of 1-2 Myr for IC 348 (Luhman et al. 2000) and 3 Myr for Taurus (White \& Ghez 2001). If we further adopt the Luhman (1999) spectral type-temperature scale for low-mass pre-main sequence objects, then theoretical evolutionary models (Baraffe et al. 1998) imply masses of $\sim 0.1-0.02 M_{\odot}$ for our M5-M8 objects. Thus, our sample spans a mass range from the stellar/sub-stellar boundary well into the brown dwarf domain (and possibly even into the planetary mass regime; Mohanty et al. 2003b).

\section{Observations and Analysis}

We obtained optical spectra of the target sample using the High Resolution Echelle Spectrometer (HIRES; Vogt et al. 1994) on the Keck I telescope on 2001 October 27 UT (CFHT-BD-Tau-2, 3 and 4) and on 2002 October 31 UT (the rest, plus a second spectrum of CFHT-3). With the 1.15" slit, the two-pixel-binned spectral resolution is $\mathrm{R} \approx 33,000$. The instrument yielded 15 spectral orders in the $6390-8700 \AA$ wavelength range, with gaps between the orders, providing a variety of features related to youth and accretion activity. For comparison to our targets and to derive $v$ sin $i$, we used $\mathrm{M}$ dwarf and $\mathrm{M}$ giant spectroscopic standards observed with the same HIRES set up. The data were reduced in a standard manner using IDL routines, as described in Basri et al. (2000).

We derived rotational velocities $(v \sin i)$ of the targets by cross-correlating with a 'spunup' template of a slowly rotating standard. Multiple spectral orders $(\sim 6)$, selected on the basis of an absence of strong telluric features, strong gravity-sensitive features, and stellar emission lines, were used in the cross-correlation analysis. Following White \& Basri (2003), we used a combination of giant and dwarf spectra for the template (see detailed discussion in Mohanty \& Basri 2003 and Mohanty et al. 2003a).

\footnotetext{
${ }^{1}$ The spectral classification of the Taurus targets by Martín et al. (2001) has been modified by Briceño et al. (2002). We adopt the latter authors' types, which are consistent with Luhman's (1999) typing scheme for the IC 348 targets.
} 


\section{Results and Discussion}

Table 1 lists the derived $v \sin i, \mathrm{H} \alpha$ equivalent widths, and $\mathrm{H} \alpha$ full widths at $10 \%$ of the peak flux for our sample. Figure 1 shows $\mathrm{H} \alpha$ line profiles in our sample. Of the ten IC 348 objects, five show broad, asymmetric $\mathrm{H} \alpha$ lines; three of those five also exhibit strong

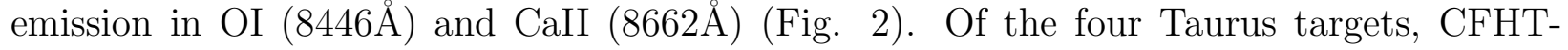
BD-Tau-3 and CFHT-BD-Tau-4 show very strong but relatively narrow $\mathrm{H} \alpha$. We have clear detections of $\mathrm{Li}(6708 \AA)$ absorption in IC348-256, 286 and 353, and marginal detections in CFHT-BD-Tau-2 and 3. The other spectra are too noisy in this region for unambiguous detection (not surprising for these very faint, red objects).

\subsection{Disk Accretion}

The shape and width of the $\mathrm{H} \alpha$ emission profile is commonly used to discriminate between accretors and non-accretors among T Tauri stars (TTS). Stars exhibiting broad, asymmetric $\mathrm{H} \alpha$ lines with equivalent width larger than $10 \AA$ are generally categorized as classical TTS (CTTS), although this threshold value varies with spectral type (e.g., Martín 1998). Recently White \& Basri (2003; hereafter WB) have suggested that a full-width $>270$ $\mathrm{kms}^{-1}$, at $10 \%$ of the peak emission, is a better empirical indicator of accretion, independent of spectral type.

By the latter criterion, two of our targets are accretors: IC348-165 (M5.25) and IC348205 (M6), with 10\% full-width > $300 \mathrm{kms}^{-1}$. Moreover, both objects show broad (FWHM >

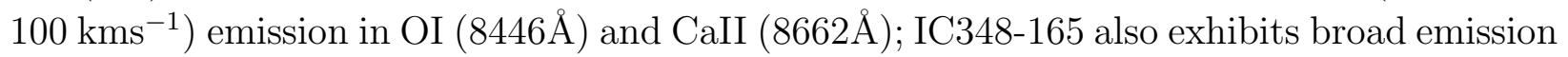

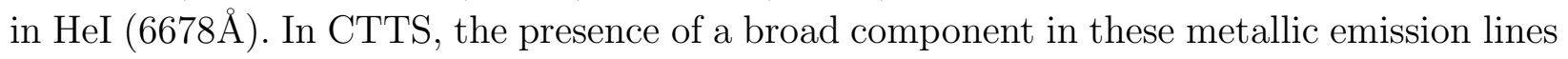
is associated with moderate to high accretion rates $\left(\dot{M} \gtrsim 10^{-8} \mathrm{M}_{\odot} / \mathrm{yr}\right)$, such as in DG Tau and DR Tau (Hessman \& Guenther 1997). WB detected these lines in CIDA-1 (M5.5) and GM Tau (M6.5), two Taurus objects at the sub-stellar boundary that also exhibit broad $\mathrm{H} \alpha$ profiles and veiling, and inferred accretion rates $\left(\sim 10^{-8.5} \mathrm{M}_{\odot} / \mathrm{yr}\right)$ similar to CTTS. In fact, Muzerolle, Hartmann, \& Calvet (1998; hereafter MHC) found a correlation between the accretion rates and the line fluxes in CaII, OI and HeI for their sample of CTTS, and argued that the broad components of these lines originate in magnetospheric infall regions.

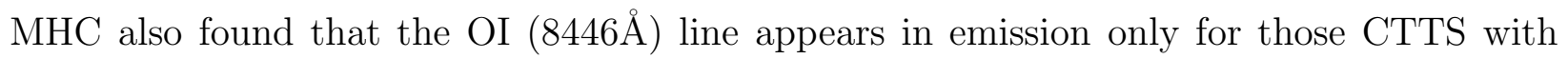
accretion rates higher than a certain threshold value $\left(\sim 10^{-8} \mathrm{M}_{\odot} / \mathrm{yr}\right)$. Thus, our detection of broad OI and CaII emission in IC348-165 and IC348-205 (as well as broad HeI in the former) is significant, and implies that some VLM objects are accreting in a fashion analogous to CTTS. 
We also find broad OI and CaII emission in a third object, IC348-415 (M6.5) (Fig.2). The results of MHC quoted above then indicate that accretion is very likely in this object as well. It is thus noteworthy that its $\mathrm{H} \alpha 10 \%$ full -width $\left(\sim 213 \mathrm{kms}^{-1}\right)$ is somewhat below the accretion cutoff $\left(270 \mathrm{kms}^{-1}\right)$ defined by WB. We suggest that the latter cutoff may need revision for VLM objects, for the following reason. The broad component of the $\mathrm{H} \alpha$ line is believed to arise in a nearly free-falling flow between the inner edge of the disk and the stellar surface (e.g., Hartmann, Hewett, \& Calvet 1994); the lower limit on the line-broadening is then set by the free-fall velocity $\left(v_{f f}\right)$. For ages $1-3 \mathrm{Myr}$ and substellar masses $(M \lesssim 0.08$ $\mathrm{M}_{\odot}$ ), evolutionary models (Baraffe et al. 1998) yield $v_{f f} \sim 75-125 \mathrm{kms}^{-1}$, implying $\mathrm{H} \alpha$ full-widths of order 150-250 $\mathrm{kms}^{-1}$, significantly less than the WB cutoff of $\sim 270 \mathrm{kms}^{-1}$. Muzerolle et al. (2001) argue that Stark broadening can increase the $\mathrm{H} \alpha$ width beyond that expected from infall alone. However, this effect does not appear important at rates below $\sim 10^{-9} \mathrm{M}_{\odot} / \mathrm{yr}$; thus in mildly accreting brown dwarfs, the $\mathrm{H} \alpha$ line-width is likely to be set by $v_{f f}$ alone. For instance, in V410 Anon 13 (a Taurus M6 object with $\dot{M} \sim 5 \times 10^{-12}$ $\mathrm{M}_{\odot} / \mathrm{yr}$ ), Muzerolle et al. (2000) adequately model $\mathrm{H} \alpha$ with no recourse to Stark broadening. These authors also show that accretion rates $\lesssim 10^{-9} \mathrm{M}_{\odot} / \mathrm{yr}$ are not expected to produce any optical veiling arising from the accretion shock. The WB cutoff, however, is defined in terms of optical veiling: only objects with veiling are adopted as accretors, and it is these that have $\mathrm{H} \alpha$ full-widths $>270 \mathrm{kms}^{-1}$. Our foregoing discussion suggests that VLM objects with very low accretion rates can fail the WB accretion test: their $\mathrm{H} \alpha$ profiles are unlikely to be Stark-broadened beyond the $v_{f f}$ full-widths, which are less than $270 \mathrm{kms}^{-1}$; furthermore, they will also not exhibit any veiling indicative of accretion. A case in point is V410 Anon 13 itself: its $\mathrm{H} \alpha 10 \%$ full-width is $\sim 250 \mathrm{kms}^{-1}$ (slightly below the WB limit), and it shows no veiling (and no CaII or OI emission either), yet is an accretor. Similarly IC348-415, which does show CaII and OI but has an $\mathrm{H} \alpha$ full-width somewhat below the WB limit, is very likely to be accreting.

Consequently, we adopt a $10 \%$ full-width $\sim 200 \mathrm{kms}^{-1}$ as our accretion cutoff. Given the rotational velocities in our sample (all $v \sin i<50 \mathrm{kms}^{-1}$ ), such a width is hard to explain by rotational broadening and/or flaring, but is in agreement with the expected $v_{f f}$. In that case, at least two other targets in our sample - IC348-382 (M5.5) and IC348-355 (M8) - are accretors as well, albeit with low accretion rates. Both show broad asymmetric $\mathrm{H} \alpha$ profiles ( $10 \%$ full-width $>200 \mathrm{kms}^{-1}$ ) associated with infall, even though the $10 \%$ width is somewhat below the WB limit and no CaII or OI emission is noticeable. If so, IC348-355 is the latest spectral type (lowest mass) accretor known to date.

Putting together now all the young objects near or below the substellar boundary ( M5 and later) with published high-resolution optical spectra, we have 4 objects in $\rho$ Ophiuchus, 
10 in IC 348, 14 in Taurus and 11 in Upper Scorpius ${ }^{2}$. Of these, optical spectral signatures of accretion are found in 1 object in $\rho$ Oph (GY 5), 5 in IC 348 (discussed above), 3 in Taurus (CIDA-1, GM Tau, V410 Anon 13) and 1 in Upper Sco (USco 75 (M6), adopting an accretion cutoff of $\sim 200 \mathrm{kms}^{-1}$ in $10 \%$ width). The vast majority of $\rho$ Oph VLM objects were inaccessible to our optical spectroscopy because of significant extinction, presumably due to circumstellar as well as interstellar material. Thus, our (small) $\rho$ Oph sample is heavily biased against possible accretors, and should not be used to estimate the accreting fraction. Considering the other three clusters, which are much less affected by this bias, we find that $\sim 50 \%$ of the VLM objects show disk accretion at an age $\lesssim 2$ Myr (IC 348), $20 \%$ at age $\sim 3$ Myr (Taurus), and $\lesssim 10 \%$ by $\sim 5$ Myr (Upper Sco). While there are uncertainties in the cluster ages, IC 348 is likely to be younger than Taurus and Upper Sco. Thus, we appear to be seeing a decrease in the fraction of accreting young sub-stellar objects with increasing age.

A decrease in brown dwarf disk frequency with age is also seen in measurements of infrared excess. While a large fraction $-\sim 60-80 \%$ - of brown dwarfs in the $\lesssim 3$ Myr-old $\rho$ Oph, IC 348, and Trapezium clusters show near-infrared excess indicative of disks, the fraction appears to be lower in the somewhat older $\sigma$ Orionis ( $\sim 5-8 \mathrm{Myr})$ and TW Hydrae ( 10 Myr) groups (Jayawardhana et al. 2003; Jayawardhana, Ardila \& Stelzer 2002; Muench et al. 2001, Liu et al. 2003). The disk fractions as measured by near-infrared excess are roughly comparable between TTS and sub-stellar objects in the same cluster. The evidence to date is consistent with similar timescales for inner disk dissipation in young brown dwarfs and low-mass stars (Jayawardhana et al. 1999; 2001; Haisch, Lada \& Lada 2001).

\subsection{Rotation and Activity}

In field $\sim \mathrm{M} 5-\mathrm{M} 8$ dwarfs, a saturation-type rotation-activity connection is seen: stars rotating above a threshold velocity $\left(\sim 5 \mathrm{kms}^{-1}\right)$ exhibit a saturated level of chromospheric $\mathrm{H} \alpha$ flux, while slower rotators show a range of (smaller) fluxes (Mohanty \& Basri 2003). We will explore this issue for our young VLM objects in a future paper, after deriving effective temperatures so that observed $\mathrm{H} \alpha$ equivalent widths can be converted to fluxes. However, we note here that the $\mathrm{H} \alpha$ widths in our sample of non-accretors are at least as large as in saturated field dwarfs of similar spectral type $(\sim 3-10 \AA)$. Indeed, in three cases - IC348-256 and CFHT-BD-Tau-3 and 4 - the equivalent widths are many times higher than usually seen

\footnotetext{
${ }^{2}$ Most from this paper and Jayawardhana, Mohanty \& Basri (2002); 9 in Taurus from WB and 1 from Muzerolle et al. (2000).
} 
in quiescent $\mathrm{M}$ dwarfs, and are reminiscent of intense chromospheric flaring. Interestingly, CFHT-BD-3 shows very strong $\mathrm{H} \alpha$ emission in two observations separated by a year. All these facts indicate that chromospheric activity levels in young VLM objects are at least as high as, and possibly significantly higher than, in older field dwarfs of similar spectral type.

\section{Concluding Remarks}

Our high-resolution optical spectra provide the most compelling evidence yet for a $\mathrm{T}$ Tauri-like accretion phase in many sub-stellar objects, qualitatively similar to that of their stellar counterparts. The lifetimes of inner disks around brown dwarfs do not appear to be vastly different from those of $\mathrm{T}$ Tauri disks. Thus, the observational evidence to date favors a similar mechanism for the formation of stellar and sub-stellar objects.

While it is too early to rule out the ejected embryo hypothesis for the origin of at least some brown dwarfs, our results do not support it: the naive expectation of signficantly shorter lifetimes for sub-stellar disks is not borne out by the data. However, current numerical simulations do not have sufficient resolution to determine whether a sub-stellar object with a small, truncated disk should be an accretor at a few million years (Bate et al. 2003). Future simulations should clarify this issue. On the observational front, longer-wavelength measurements with the Space InfraRed Telescope Facility and/or the Stratospheric Observatory

For Infrared Astronomy could constrain the sizes of brown dwarf disks and thus determine if they are truncated as expected in the ejection scenario. Other important insights could come from investigating even younger objects, namely embedded (proto-) brown dwarfs, with high-resolution near-infrared spectroscopy.

We would like to acknowledge the great cultural significance of Mauna Kea for native Hawaiians, and express our gratitude for permission to observe from its summit. We also thank the Keck Observatory staff for their outstanding assistance over the past several years. This work was supported in part by NSF grants AST-0205130 to R.J. and AST-0098468 to G.B. S.M. acknowledges the support of a SIM-YSO postdoctoral fellowship.

\section{REFERENCES}

Baraffe, I., et al. 1998, A\&A, 337, 403

Basri, G., et al. 2000, ApJ, 538, 363

Bate, M.R., Bonnell, I.A., \& Bromm, V. 2002, MNRAS, 332, L65 
Bate, M.R., Bonnell, I.A., \& Bromm, V. 2003, MNRAS, 339, 577

Briceño, C., et al. 2002, ApJ, 580, 317

Haisch, K.E.,Jr., Lada, E.A., \& Lada, C.J. 2001, 553, L153

Hartmann, L., Hewett, R., \& Calvet, N. 1994, ApJ, 426, 669

Hessman, F.V. \& Guenther, E.W. 1997, A\&A, 321, 497

Jayawardhana, R. et al. 1999, ApJ, 521, L129

Jayawardhana, R. et al. 2001, ApJ, 550, L197

Jayawardhana, R., Ardila, D.R., \& Stelzer, B. 2002, in Brown Dwarfs, ed. E. L. Martín, San Francisco: Astronomical Society of the Pacific

Jayawardhana, R., Mohanty, S., \& Basri, G. 2002, ApJ, 578, L141

Jayawardhana, R. et al. 2003, AJ, submitted

Joy, A.H. 1945, ApJ, 102, 168

Liu, M.C., et al. 2003, ApJ, 585, 372

Luhman, K.L. 1999, ApJ, 525, 466

Luhman, K.L., et al. 2000, ApJ, 540, 1016

Luhman, K.L., et al. 2003, ApJ, submitted

Martín, E.L., et al. 2001, ApJ, 561, L195

Mohanty, S. \& Basri, G., 2003, ApJ, 583, 451

Mohanty, S., et al., 2003a, ApJ, submitted

Mohanty, S., Jayawardhana, R., Basri, G., 2003b, ApJ, submitted

Muench, A.A., et al. 2001, ApJ, 558, L51

Muzerolle, J., Hartmann, L., \& Calvet, N. 1998, AJ, 116, 455 [MHC]

Muzerolle, J., et al. 2000, ApJ, 545, L141

Muzerolle, J., Calvet, N. \& Hartmann, L. 2001, ApJ, 550, 944

Padoan, P. \& Nordlund, A. 2003, ApJ, in press

Reipurth, B. \& Clarke, C. 2001, AJ, 122, 432

Vogt, S.S., et al. 1994, Proc. SPIE, 2198, 362

White, R. J., \& Basri, G. 2003, ApJ, 582, 1109 [WB]

White, R.J. \& Ghez, A.M. 2001, ApJ, 556, 265

This preprint was prepared with the AAS IATEX macros v5.0. 

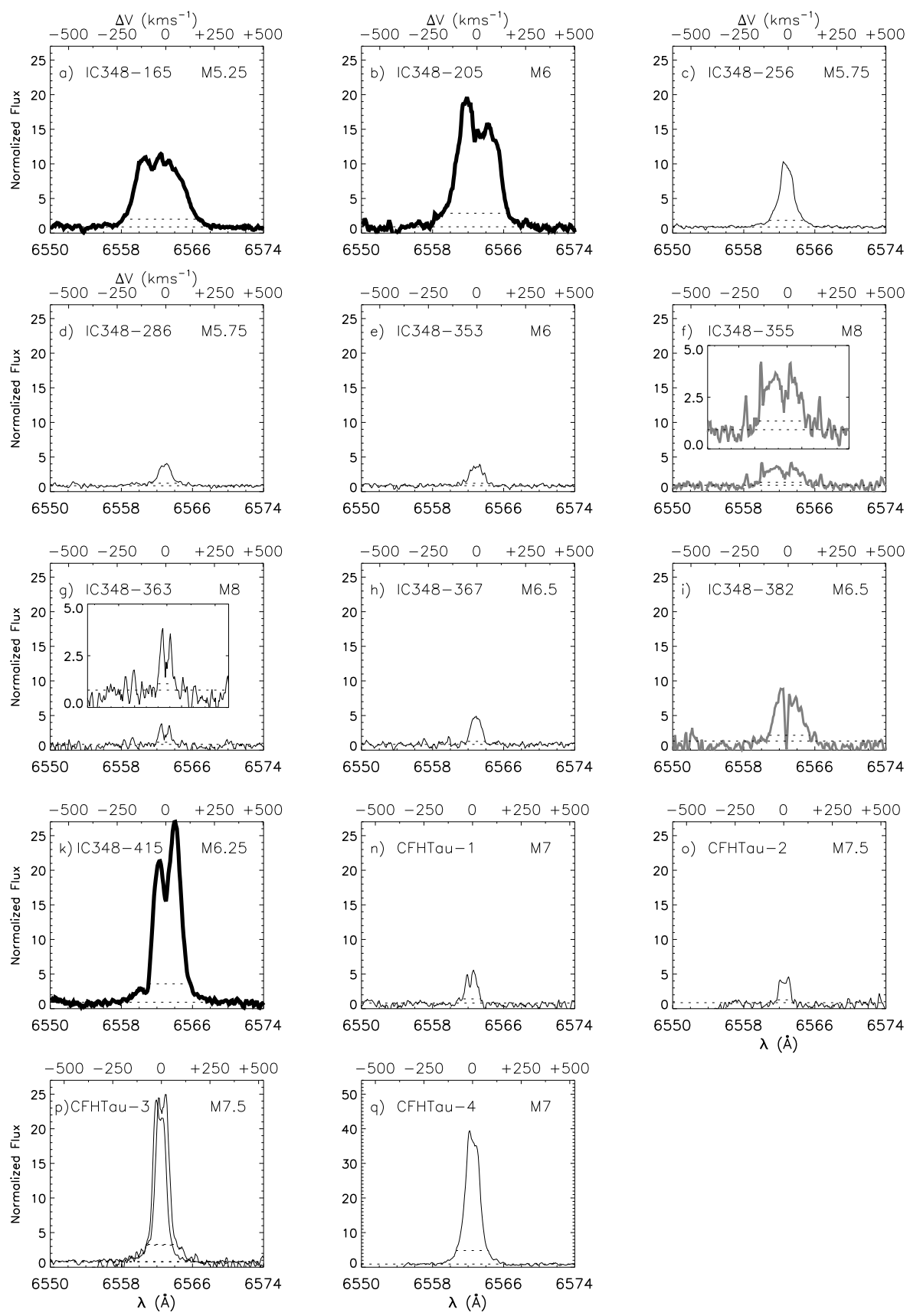

Fig. 1. - $\mathrm{H} \alpha$ line profiles of the target sample. Spectra shown have been smoothed by a 3-pixel boxcar; continuum and full width at $10 \%$ of the peak levels are marked by dotted lines. Thick black lines indicate accretors with broad $\mathrm{H} \alpha$ as well as CaII and OI emission; grey indicates probable accretors, based on the $\mathrm{H} \alpha$ profile-shape and $10 \%$ full-width. Insets zoom in on objects with low peak-flux and noisy continuua, to clearly show the $\mathrm{H} \alpha$ detection. For CFHT-3, two spectra are shown, separated by a year; note the similarly strong emission both times. For CFHT-4, note the change in Y-axis scale; the peak flux in this object is much higher than in any other target in our sample. 

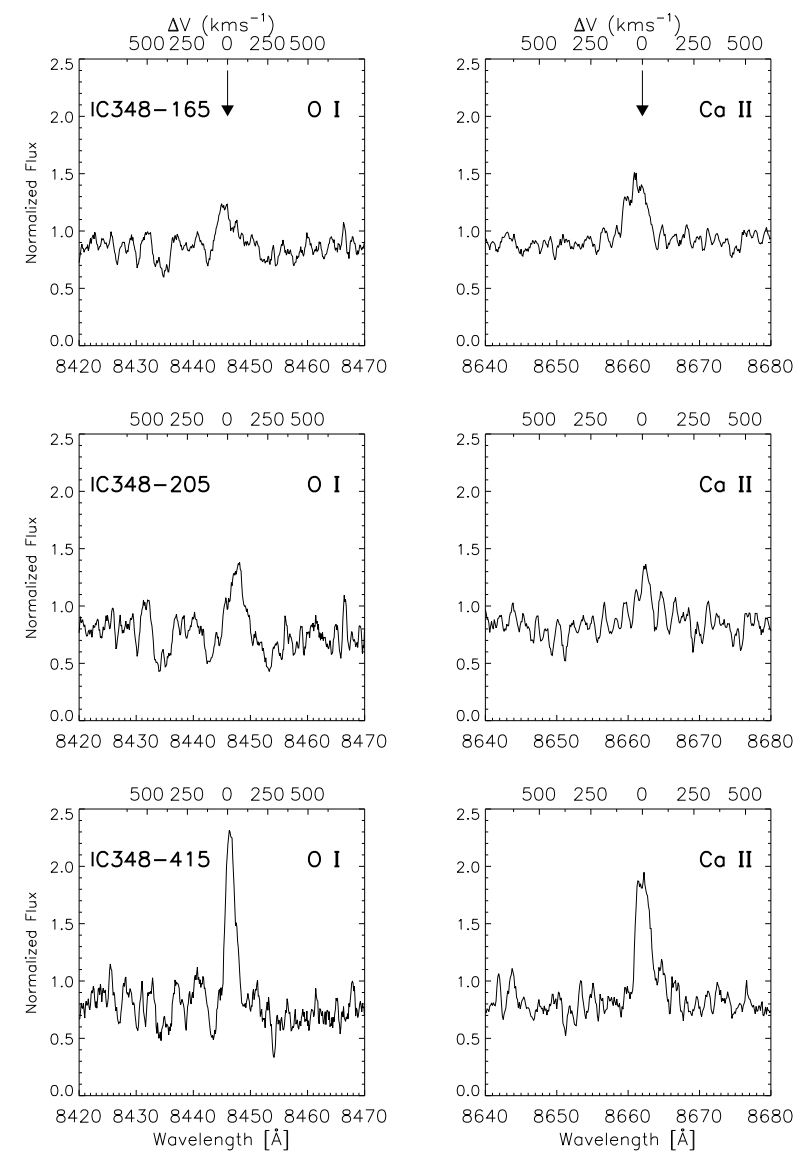

Fig. 2.- Line profiles of OI $(8446 \AA)$ and CaII $(8662 \AA)$ in three IC 348 objects. Arrows indicate line-center. These spectra have been smoothed by a 4 -pixel boxcar. 
Table 1.

\begin{tabular}{|c|c|c|c|c|c|c|}
\hline Object & Sp Type & $\begin{array}{l}\mathrm{v} \sin \mathrm{i} \\
\left(\mathrm{kms}^{-1}\right)\end{array}$ & $\begin{array}{c}\mathrm{H} \alpha \mathrm{EW}^{\mathrm{b}} \\
(\AA)\end{array}$ & $\begin{array}{c}\mathrm{H} \alpha 10 \% \mathrm{FW} \\
\left(\mathrm{kms}^{-1}\right)\end{array}$ & $\begin{array}{l}\text { OI } \mathrm{EW}^{\mathrm{b}, c} \\
(\AA)\end{array}$ & $\begin{array}{c}\text { CaII EW } \\
(\AA)\end{array}$ \\
\hline IC348-165 & M5.25 & $19 \pm 2$ & $66 \pm 6$ & 389 & 1.1 & 1.8 \\
\hline IC348-205 & M6 & $6 \pm 2$ & $93 \pm 9$ & 338 & 2.4 & 1.5: \\
\hline IC348-256 & M5.75 & $9 \pm 2$ & $23 \pm 2$ & 180 & - & - \\
\hline IC348-286 & M5.75 & $19 \pm 3$ & $6.9 \pm 0.7$ & 148 & - & - \\
\hline IC348-353 & M6 & $25 \pm 3$ & $5.8 \pm 0.6$ & 117 & - & - \\
\hline IC348-355 & M8 & 45: & $10.3 \pm 1$ & 235 & - & - \\
\hline IC348-363 & M8 & $14 \pm 3$ & 3.8: & 82: & - & - \\
\hline IC348-367 & M5.75 & $20 \pm 3$ & 6.8: & 92: & - & - \\
\hline IC348-382 & M5.5 & 10: & $15 \pm 2$ & 208 & - & - \\
\hline IC348-415 & M6.5 & $<5$ & $80 \pm 8$ & 213 & 3.6 & 3.0 \\
\hline CFHT-BD-Tau-1 & M7 & $7 \pm 3$ & $7.4 \pm 1.5$ & 85 & - & - \\
\hline CFHT-BD-Tau-2 & M7.5 & 8: & $11 \pm 1$ & 80 & - & - \\
\hline CFHT-BD-Tau-3 & M7.75 & $12 \pm 2$ & $50 \pm 4 / 65 \pm 1$ & $136 / 153$ & - & - \\
\hline CFHT-BD-Tau-4 & M7 & 11: & $69 \pm 4$ & 175 & - & - \\
\hline
\end{tabular}

aSpectral types for IC 348 sources are from Luhman et al. (2003; for 367, 382 and 415) and Luhman (1999; for others). Spectral type for CFHT-BD-Tau-1 is from Martín et al. (2001). Spectral types for remaining CFHT Taurus sources are from Briceño et al. (2002), who use the same classification scheme as Luhman (1999) and somewhat different from Martín et al. (2001). For CFHT-2 and 3, their spectral types are respectively 0.5 and 1.25 subclasses earlier than those of Martín et al. (2001).

${ }^{\mathrm{b}}$ Pseudo-equivalent width; the plethora of molecular lines make it impossible to determine the true continuum in these stars.

${ }^{\mathrm{c}}$ For OI and CaII, large absorption troughs near the emission lines (see Fig. 2) make line-width determination difficult; our errors are $\sim \pm 15 \%$. 